\title{
Guessing the Texture of Magnetic Samples Assisted by Aharonov-Bohm Effect
}

\author{
C. A. Valagiannopoulos, ${ }^{1}$ A. Alù ${ }^{2}$, A. Dimakis ${ }^{2}$ and E. A. Marengo ${ }^{3}$ \\ ${ }^{1}$ Department of Physics, School of Science and Technology, \\ Nazarbayev University, 53 Qabanbay Batyr Ave, Astana, KZ-010000, Kazakhstan \\ 2 Department of Electrical and Computer Engineering, \\ University of Texas at Austin, 1616 Guadalupe St., Austin, TX-78712, U.S.A. \\ 3 Department of Electrical and Computer Engineering, \\ Northeastern University, 360 Huntington Ave, Boston, MA-02115, U.S.A.
}

\begin{abstract}
Aharonov-Bohm (AB) effect is a very significant phenomenon of quantum mechanics. It demonstrates the significance of electromagnetic potentials (proportional to fluxes) against the electromagnetic fields through the simple paradigm of two electron beams (e-beams). Even though they are both traveling through a null field space, their wave functions are not equal: the difference is in their phases and it is related to the magnetic flux through the area defined by their trajectories. Inspired by this fact, we formulate an inverse problem for the magnetic permeability of a bio-chemical sample positioned inbetween two magnets. The data can be taken by measuring the phase difference between the wave functions of two split e-beams with parallel trajectories passing through the samples. The case of only radial dependence of magnetic permeability is fully solved and certain insights are provided for the arbitrary radial/azimuthal distributions.
\end{abstract}

Index Terms: Aharonov-Bohm Effect, Inverse Problem, Magnetic Permeability

\section{INTRODUCTION}

Guessing the distribution of a feature characterizing a sample without destroying it constitutes a challenging task that is being examined for decades. Such a field has multiple applications in cell chemistry, molecular biology, medicine and pharmacy. Significant research has been performed on optical coherent tomography [1], confocal microscopy [2], and real-time in vivo optical interferometric imaging [3]. In the same optical context, multiple scattering has been found to allow the tomographic reconstruction of angular resolved scattering distributions [4]. Tomographic methods can also be used to reconstruct the quantum state of a system [5], and in this context compressed sensing has been successfully employed for quantum state imaging [6]. In fact, the estimation of quantum wave probability functions has become recently feasible through compressed sensing methods even for the demanding case of fermions [7].

Here, we propose a new approach to e-beam tomography based on the Aharonov-Bohm (AB) effect [8]. We use a pair of electron beams (e-beams) to determine the features of an inhomogeneous magnetic material distributed within a cylindrical volume and provide a more effective imaging [9] technique than previous attempts employing $\mathrm{AB}$ effect for inverse scattering [10], [11]. The proposed AB tomography is based on the estimation of the local magnetic flux density through an inhomogeneous object, through which one can indirectly invert its magnetic properties. As part of the proposed method, we consider the use of compressed sensing methods to optimize the inversion algorithm and minimize the required number of measurements to resolve the object of interest.

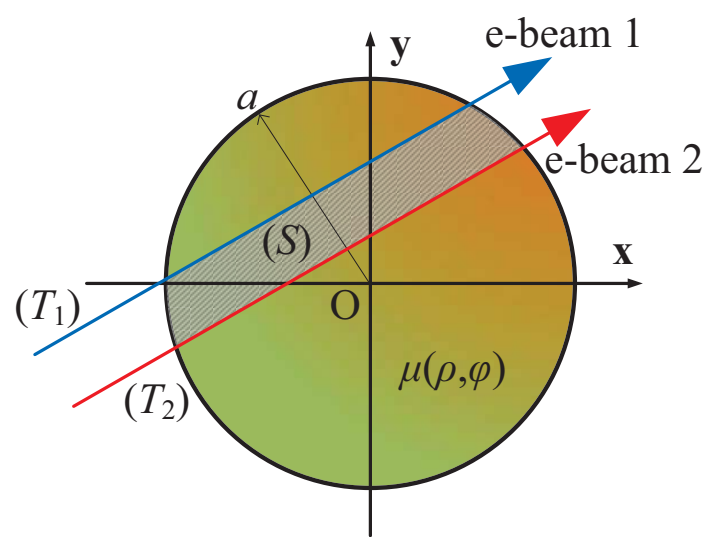

Fig. 1. The physical configuration of the considered structure. An unknown arbitrary distribution of magnetic permeability $\mu(\rho, \phi)$ existing within a cylinder of radius $a$, is estimated with use of two parallel e-beams ( 1 and 2 ) defining an area $(S)$. A single electron wavepacket is split into two alternative paths (e-beam 1 and e-beam 2) for the electron.

\section{Aharonov-Bohm (AB) EFFect}

In classical electromagnetic theory, the potentials are only intermediate quantities for the computation of the electric and magnetic fields. Only the fields, not the potentials, are usually regarded as having real physical significance. In contrast, in quantum theory, the potentials can have under certain circumstances even a more fundamental role than the corresponding fields. For example, Schrödingers wave function of an electron $e$ following a trajectory $(T)$ into three-dimensional space is well known to be of the form: $\Psi(r, t)=\exp \left[\frac{j e}{\hbar} \int_{(T)} \mathbf{A} \cdot d \mathbf{l}\right] Y(r, t)$ where $\mathbf{A}$ is the magnetic vector potential, $e$ is the electron charge, $\hbar$ is the reduced Planckian constant, and $Y$ is a determinable reference wave 
function (which corresponds, for example, to the special case of vanishing magnetic potential along the relevant trajectory).

The potential-dependent phase term in the expression for the wavefunction has physically measurable effects that can be sensed, e.g., via double slit interference experiments. In the following, we focus on the familiar experimental setting in which a coherent e-beam is split into two beams following alternative paths $\left(T_{1}\right)$ and $\left(T_{2}\right)$ which are combined at a given point (where a sensor can be placed). In this scenario, the respective wavefunction at the sensor position is defined by the interference of the split beam wavefunctions which is governed in turn by the phase shift $\frac{e}{\hbar}\left[\int_{\left(T_{2}\right)} \mathbf{A} \cdot d \mathbf{l}-\int_{\left(T_{1}\right)} \mathbf{A} \cdot d \mathbf{l}\right]$. The latter phase shift is equal to the integral along the closed loop formed by one of the paths (in the direction from the split point to the detector) and the other (returning) path (from the detector back to the split point), thus the relevant phase shift is governed by the relevant closed loop integral (or cochain) of the magnetic vector potential. This physically measurable effect of the potential can occur even if the electromagnetic field vanishes identically along the relevant electron paths so long as the respective magnetic vector potential cochain is nonzero. This is a manifestation of the well-known AharonovBohm (AB) effect, a modern cornerstone of quantum mechanics, which was first reported in 1959 [8].

The simplest example is given by the magnetostatic solenoid in cylindrical coordinates $(\rho, \phi, z)$ whose exterior electromagnetic field is null $(\mathbf{E}=\mathbf{0}=\mathbf{B})$, while the magnetic vector potential is nonzero (behaves like $\mathbf{A} \sim \hat{\phi} / \rho$. In the following we exploit the measurable quantum mechanical phase shift of split e-beams for the determination of the magnetic properties of a sample under study. In this context, we broadly interpret the $\mathrm{AB}$ effect as the key mechanism behind the above split electron phase shift due to nonzero magnetic potential cochains, regardless of the vanishing or nonvanishing nature of the fields in the relevant region. Thus the proposed $\mathrm{AB}$ effect tomography applies under rather arbitrary electrodynamic conditions and can, for instance, be applied to determine the material properties of the sample at any frequency of interest.

\section{CONCEPT FORMULATION}

The Aharonov-Bohm effect is due to the wave-nature of the electrons in the quantum level: their behavior (wave functions $\Psi$ and trajectories $\left(T_{1}\right)$ and $\left(T_{2}\right)$ of e-beams) is not affected only by their local characteristics (electromagnetic field $\mathbf{E}, \mathbf{H}$ along their paths) but also by the entire structure as a whole (magnetic flux expressed via magnetic vector potential). Therefore, the study of the behavior of e-beams can give us information about the texture of the structure. In the following, we exploit the quantum-mechanical phase shift of split ebeams to reconstruct the magnetic properties of a sample. The setup of the proposed system is depicted in Fig. 1, where a cylinder of radius a is filled with material of arbitrary magnetic permeability $\mu(\rho, \phi)$ whose estimation is the major objective of this work. The structure is placed into vacuum (free-space permeability $\mu_{0}$ ) and is fed by a $\hat{\mathbf{z}}$ polarized magnetostatic

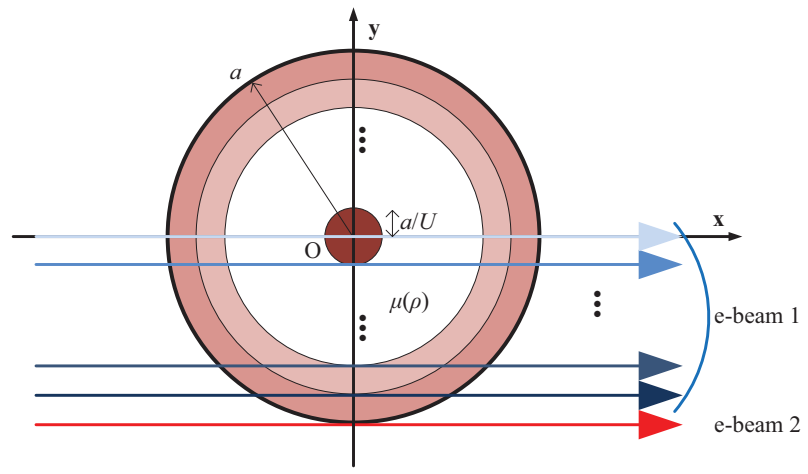

(a)

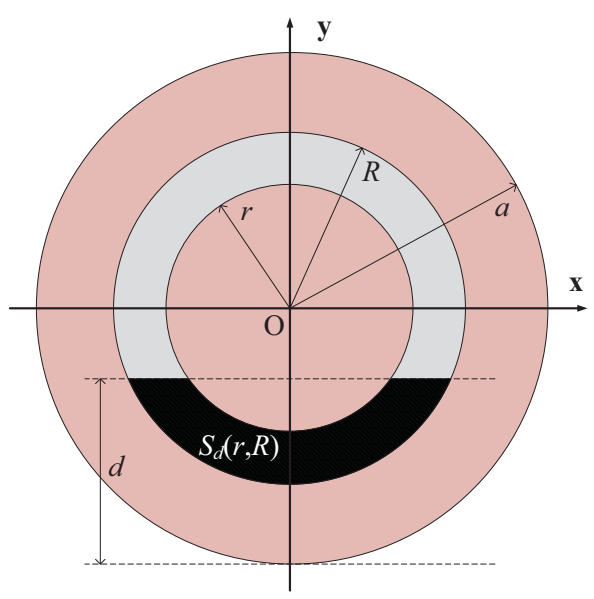

(b)

Fig. 2. (a) The $1 \mathrm{D}$ setup of radially changing permeability $\mu(\rho)$ for $0<$ $\rho<a$. The first beam is scanning the cylindrical volume by changing its vertical position, while the second one is kept fixed. (b) The area $S_{d}(r, R)$ defined by two concentric cells of radii $r$ and $R>r$ exiting below a straight line with distance $d$ from the external surface of radius $a>R$.

induction $\mathbf{B}$ for $\rho<a$ which corresponds to an azimuthal ( $\hat{\phi}$-directed in the case of homogeneous excitation) magnetic vector potential A. Under this excitation, one wavepacket is split into two alternative paths (e-beam 1 and e-beam 2) for the electron.

The two e-beams following parallel straight lines trajectories lying (for simplicity) at the same constant-z plane are passing through the cylindrical permeability distribution. If one measures the difference of phase between the wave functions $\left\{\Psi_{1}, \Psi_{2}\right\}$ of the two e-beams, it is proportional to the magnetic flux through the area $(S)$ defined by the cross section of the two parallel beams and the cylinder, namely:

$$
\Phi(\mu)=\oint_{(\partial S)} \mathbf{A} \cdot d \mathbf{l}=\iint_{(S)} \mathbf{B} \cdot d \mathbf{s}=\iint_{(S)}(H \mu) \cdot d s
$$

where $H$ is the magnetic field. Now, by considering several beam pairs, corresponding to different directions, it is possible to gather enough information about the magnetic flux associated to several cross sections of the object which allows one to estimate the magnetic flux crossing through 
smaller (intersection-defined) cross sections of the object. If the objective is to measure the permeability $\mu$ of a sample, then one can impose a known magnetic field, e.g., via a magnet or solenoid in the vicinity of the sample. Then from the reconstruction of the local magnetic flux $\Phi$ one can in turn estimate the sought-after permeability distribution $\mu$.

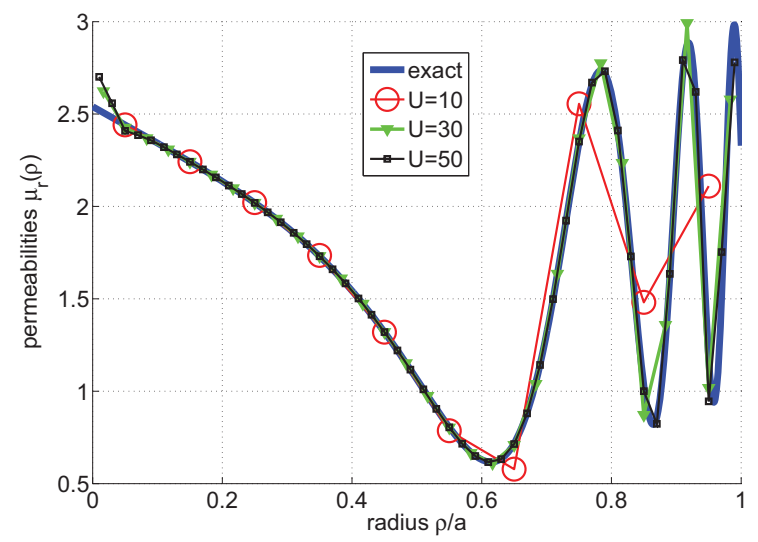

(a)

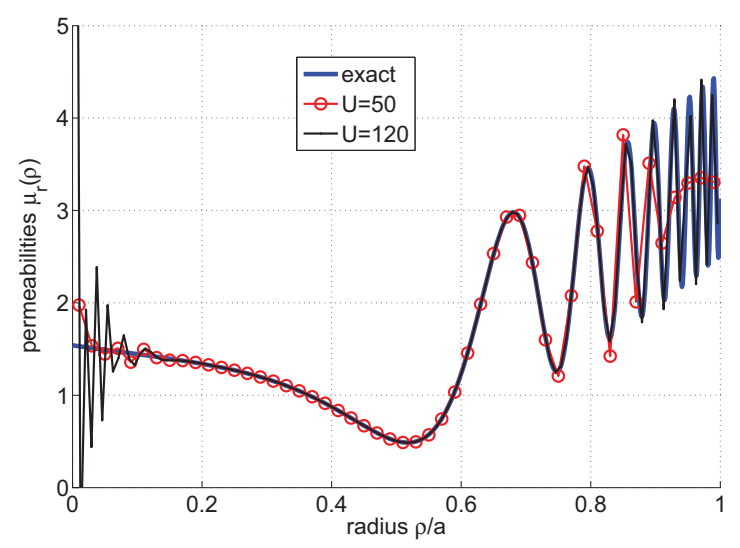

(b)

Fig. 3. The $1 \mathrm{D}$ exact permeability distributions $\mu(\rho) / \mu_{0}$ compared with the approximate ones for various discretization number of layers $U$ for: (a) $g=5$ and (b) $g=30$.

\section{NUMERICAL RESUltS}

When the magnetic texture of the structure is only radially changing, namely the magnetic permeability is a function $\mu=\mu(\rho)$, the application of proposed approach is straightforward (one-dimensional, 1D). For simplicity, we consider with spatially homogeneous magnetic field $\mathbf{B}=\hat{\mathbf{z}} \mu_{0} H_{0}$ in order for the magnetic flux measurement to be indicative of the local value of the permeability. If the one beam is kept fixed outside of the structure and the other is moving gradually (by covering distance $\Delta \rho$ each time) towards the core of the cylinder, the measurements of the corresponding magnetic flux lead to a well-defined linear system with respect to the permeability values. There is no scattering field component due to the presence of the cylinder since the field is static and parallel to the axis of the cylinder so no Born approximation is required as in the corresponding electrodynamics problems [12]. With reference to Fig. 2(a), the unknown distribution $\mu=\mu(\rho)$ is step approximated by the values $\mu_{u}=\mu((u+1 / 2) a / U)$ for $u=0, \cdots,(U-1)$, where here $\Delta \rho=a / U$. If the measurements of the magnetic flux through the area $(S)$ defined by the fixed beam 2 and the moving beam 1 are included in a vector $\boldsymbol{\Phi}$, a $U \times U$ linear system $\boldsymbol{\Sigma} \cdot \boldsymbol{\mu}=\frac{1}{H_{0}} \boldsymbol{\Phi}$ with respect to the unknown permeabilities $\boldsymbol{\mu}=\left[\mu_{1} \cdots \mu_{U}\right]^{T}$ is formulated with a matrix $\boldsymbol{\Sigma}=\left[\Sigma_{u v}\right]$ ( $u$ is the number of rows and $v$ the number of columns), whose elements are given by:

$$
\Sigma_{u v}=\left\{\begin{array}{cl}
S_{u \Delta \rho}((v-1) \Delta \rho, v \Delta \rho) & u \geq v \\
0 & u<v
\end{array},\right.
$$

where the area $S_{d}(r, R)$ is defined in Fig. 2(b) and is analytically determinable. The matrix $\boldsymbol{\Sigma}$ is bottom triangular and thus the numerical inversion is implemented in an accurate and robust way.

To demonstrate the feasibility of our approach, we consider the following permeability profile:

$$
\mu(\rho)=\mu_{0}\left[2+\cos \left(\left(\frac{\rho}{a}\right)^{2} g\right)\right],
$$

where the magnetic flux vector can be found analytically. In Figs. 3, two cases for different variation are considered and multiple estimations for the permeability profiles are given for different $U$ together with the exact one. The three-dimensional distributions across the cylindrical disk $0<\rho<a$ are depicted in the respective insets. In Fig. 3(a) we regard a slow radial variation $(g=5)$ and we notice that the coincidence is remarkable for $U=12$ discretization layers, while even for $U=3$, the main variation has been captured. The situation is different in Fig. 3(b), where the permittivity change is more rapid and finer chopping $(U=90)$ of the cylinder with use of the two e-beams is required. Note that with a coarse discretization of $U=10$, fails completely to predict the rapid fluctuations for $\rho>a / 2$.

When the unknown magnetic texture is dependent on both the radial $\rho$ and the azimuthal $\phi$ coordinate, more advanced techniques of compressed sensing are required. In particular, the sample is "chopped" into pixels and the unknown distribution is approximated by discrete values. The direction of e-beams and their entrance points are randomly selected. The spatial modulation of the background field helps in most cases to achieve a result of similar quality but faster. Furthermore, a transformation of the variables (dictionary) is sometimes required so that they get expressed in an alternative base where the unknown permeability distribution $\mu(\rho, \phi)$ is sparse. The thorough consideration of that two-dimensional (2D) case would be the next step of our research on the topic.

\section{Conclusions}

The trajectories of electron beams are affected not only by the local fields along them but also by the fluxes through the 
area surrounding them (Aharonov-Bohm effect). Therefore, the wave functions of the wavepackets can carry information about the magnetic flux and accordingly (for known background field) the magnetic permeability of the region where they pass. In this way, we formulate an inverse problem which is analytically solvable for one-dimensional samples. The twodimensional problem is currently under consideration and various alternative techniques can be implemented to tackle with it.

\section{REFERENCES}

[1] D. Huang, E. A. Swanson, C. P. Lin, J. S. Schuman, W. G. Stinson, W. Chang, M. R. Hee, T. Flotte, K. Gregory, C. A. Puliafito, and J. G. Fujimoto, "Optical coherence tomography", Science, vol. 22, pp. 1178$1181,1991$.

[2] J. Sharpe, U. Ahlgren, P. Perry, B. Hill, A. Ross, J. Hecksher-Sorensen, R. Baldock, and D. Davidson, "Optical projection tomography as a tool for 3D microscopy and gene expression studies", Science, vol. 296, pp. $541-545,2002$

[3] A. Ahmad, N. D. Shemonski, S. G. Adiel, H.-S. Kim, W.-M. W. Hwu, P. S. Carney and S. A. Boppart, "Real-time in vivo computed optical interferometric tomography", Nature Photonics, vol. 7, pp. 444-448, 2013.

[4] P. Modregger, M. Kagias, S. Peter, M. Abis, V. A. Guzenko, C. David, and M. Stampanoni, "Multiple scattering tomography", Physical Review Letters, vol. 113, no. 020801, 2014.

[5] J. Rehacek, Z. Hradil, Z. Bouchal, R. Celechovsky, I. Rigas, "Full tomography from compatible measurements", Physical Review Letters, vol. 103, no. 250402, 2009.

[6] D. Gross, Y.-K. Liu, S. T. Flammia, S. Becker, and J. Eisert, "Quantum state tomography via compressed sensing", Physical Review Letters, vol. 105, no. 150401, 2010.

[7] T. Jullien, P. Roulleau, B. Roche, A. Cavanna, Y. Jin and D. C. Glattli, "Quantum tomography of an electron", Nature, vol. 514, pp. 603-607, 2014.

[8] Y. Aharonov and D. Bohm, "Significance of electromagnetic potentials in the quantum theory", Physical Review, vol. 115, pp. 485-491, 1959.

[9] C. A. Valagiannopoulos, "A new inverse formula based on the Taylor expansion of the scattering integral", Electromagnetics, vol. 30, pp. 565573, 2010.

[10] P. A. Midgley and R. E. Dunin-Borkowski, "Electron tomography and holography in materials science", Nature Materials, vol. 8, pp. 271-280, 2009.

[11] I. Neder, N. Ofek, Y. Chung, M. Heiblum, D. Mahalu, and V. Umansky, "Interference between two indistinguishable electrons from independent sources", Nature, vol. 448, pp. 333-337, 2007.

[12] C. A. Valagiannopoulos, "A novel methodology for estimating the permittivity of a specimen rod at low radio frequencies", Journal of Electromagnetic Waves and Applications, vol. 4, pp. 631-640, 2010. 